${ }^{1}$ ГУЗ областной клинический онкологический диспансер (Ульяновск, Россия)

${ }^{2}$ Oregon Health and Science University (Oregon, USA)

${ }^{3}$ Banner Health MD Anderson Cancer Center (Arizona, USA)

\section{6 ГОД:}

\section{НОВОЕ В ЛУЧЕВОЙ ТЕРАПИИ ЗЛОКАЧЕСТВЕННЫХ ОПУХОЛЕЙ}

Н.В. Деньгина ${ }^{1}$, Т.В. Митин ${ }^{2}$, А.О. Лихачёва ${ }^{3}$

2016: NEW DIRECTIONS IN RADIATION THERAPY

Н.В. Деньгина ${ }^{1}$ Кандидат медицинскй наук, радиологическое отделение, ГУЗ областной клинический онкологический диспансер, 432017, Россия, Ульяновск, ул.12 Сентября, 90. Тел.: 8 (8422) 327547 , E-mail: indigo171201@yaboo.com.

T.B. Митин ${ }^{2}$ Доктормедицинских наук.

A.О. Лихачёва Доктор медицинских наук, магистр общественного здравоохранения.

N.V. Dengina ${ }^{1}$ Candidate of Medicine, Radiotherapy Department, Regional clinical oncological dispensary, 432017, Russia, Ulyanovsk, 12th of September Street, 90. Phone: 8 (8422) 327547 E-mail: indigo171201@yaboo.com

T.V. Mitin ${ }^{2}$

Doctor of Medicine, Department of Radiation Medicine, Oregon Health and Science University, 97239, USA, OR, Portland, SW Sam Jackson Park Rd, 3181. E-mail:mitin@obsu.edu, Phone: +15036814200.

A.O. Likhacheva ${ }^{3}$ Doctor of Medicine, Master of Public Health, Department of Radiation Oncology, Banner Health MD Anderson Cancer Center, 85234, USA, AZ, Gilbert, 2946 E Banner Gateway Dr. Phone: 480-256-4500.

В данном обзоре рассматриваются современные подходы к назначению адъювантной лучевой терапии у больных раком молочной железы в зависимости от ответа на неоадъювантную химиотерапию, а также освещены результаты крупных исследований по раку предстательной железы, опубликованных в 2016 году, способных изменить традиционные подходы к назначению лучевой терапии у этих категорий больных. Особое внимание уделено возрастающей актуальности комбинации облучения с таргетными препаратами в лечении больных с опухолями различных локализаций, а также возможностям лучевой терапии при воздействии на опухоли печени и желчных протоков, традиционно считающихся радиорезистентными.

ключевые слова: лучевая терапия, рак молочной железы, рак предстательной железы, таргетная терапия, абскопальный эффект, гепатоцеллюлярная кариинома, внутрипеченочная холангиокарцинома. 
This article focuses on the approaches to the adjuvant radiotherapy for breast cancer patients in accordance with the neoadjuvant chemotherapy response and also highlights the results of major studies on prostate cancer published in 2016, which are able to change the traditional approach to the radiotherapy administration in these categories of patients. Particular attention is given to the increasing relevance of irradiation combined with targeted agents for the treatment of patients with various tumors, as well as to the opportunities of radiotherapy for hepatocellular carcinoma and bile tract tumors, traditionally considered radioresistant.

Keywords: radiotherapy, breast cancer, prostate cancer, targeted therapy, abscopal effect, bepatocellular carcinoma, intrabepatic cholangiocarcinoma.

$\mathrm{Y}$ тверждать, что прошедший 2016 год, традиционно нелегкий високосный год потрясений, природных катаклизмов и человеческих потерь, привнес нечто кардинально новое в лучевую терапию, было бы неправильно. Однако, на наш взгляд, во многом он явился годом переосмысления имеющихся многочисленных фактов в отношении лечения опухолей различных локализаций, отчасти - неким возвратом к «старым песням о главном», что, возможно, изменит в дальнейшем традиционные подходы, существовавшие годами. В то же время, продолжается поиск новых путей как изолированного локального (лучевого), так и комбинированного воздействия на опухоли, в лечении которых пока не удалось достичь удовлетворяющих нас всех результатов.

\section{Взгляд на традиционные подходы под особым углом}

\section{1. Как ответ на неоадъювантную химиотера- пию.может повлиять нарешение о необходимости назначения адвювантной лучевой терапии после мастэктомии при раке молочной жслезы}

Роль послеоперационной лучевой терапии (ЛТ) после неоадъювантной химиотерапии (ХТ) с последующей мастэктомией - тема не новая, но заставляющая в последние годы взглянуть на себя в относительно новом контексте. Говорить подробно о роли ЛТ в лечении больных раком молочной железы (РМЖ), ссылаясь на многочисленные крупные исследования, в этой статье мы не будем, подчеркнув, тем не менее, что добавление адъювантного облучения в план лечения после мастэктомии или органосохраняющего лечения позволяет снизить риск локального рецидива, риск смертности от РМЖ и улучшить показатели общей выживаемости [1-6].

Согласно наиболее поздней версии рекомендаций NCCN 2016 года по ЛT после радикальной мастэктомии, сформулированных практикующими экспертами в этой области, нет разногласий в отношении больных с массивным поражением л/узлов (4 и более) - они должны получать ЛТ. В случаях с опухолями менее 5 см и негативными л/узлами эксперты однозначно против ЛТ. Ситуация с 1-3 л/узлами менее ясная и вызывает немалое количество дискуссий, но для таких больных большинство экспертов рекомендуют всерьез рассматривать необходимость проведения ЛТ после мастэктомии, опираясь на ряд дополнительных весомых факторов риска (массивная лимфоваскулярная инвазия, позитивный край резекции, низкая степень дифференцировки опухоли, молодой возраст пациентки, агрессивный молекулярный подтип опухоли). Сомнения в отношении необходимости назначения ЛТ после мастэктомии при ограниченном поражении л/узлов (1-3) также поддерживаются данными нескольких ретроспективных исследований и, в большей степени, ретроспективным совокупным анализом 5 крупных американских исследований, каждое из которых не подразумевало проведение ЛТ после радикальной операции [7]. Анализ результатов лечения почти 6000 пациенток показал относительно небольшой риск локального рецидива (8\% за 10 лет) для больных с 1-3 позитивными л/узлами. Тот же автop, Alphonse Taghian, в последующем исследовании 2006 года продемонстрировал, что для Т2N0 после мастэктомии и системной терапии риск рецидива также низок (7\%) и без лучевой терапии [8].

В совместных рекомендациях трех профессиональных онкологических сообществ ASCO, ASTRO и ASSO от 2016 года [9] эксперты обозначили категорию больных с T1-2N1, которым после радикальной мастэктомии, скорее всего, не понадобится адъювантное облучение: пациентки в возрасте старше 40 лет с сопутствующей соматической патологией, с единичным обнаруженным лимфоузлом, пораженным микрометастазом, при отсутствии лимфоваскулярной инвазии, при высокой степени дифференцировки опухоли и высокой гормональной чувствительности, а также что явилось новым - при условии существенного ответа на неоадъювантную ХТ. Вот тут мы и переходим к обсуждению, насколько ответ на ХТ может влиять на решение о назначении адъювантной ЛТ. До сих пор общее представление по данному вопросу подразумевало игнорирование информации об ответе на ХT с рекомендацией назначения адъювантной ЛТ на основе изначальных клинических данных, как если бы пациентка сначала прошла через радикальную мастэктомию.

Изначальная идея неоадъювантной ХТ при раке молочной железы состоит в том, что более раннее системное воздействие способствует более своевременной эрадикации микрометастазов и, следовательно, улучшению показателей общей выживаемости. К сожалению, рандомизированное исследование NSABP B18 не показало разницы в результатах после 
адъювантной и неоадъювантной ХТ [10]. Однако почти $80 \%$ больных продемонстрировали клинический эффект: половина - частичный, половина - полный, и у $13 \%$ отмечен полный патоморфологический ответ, что подразумевает более высокий шанс сохранения органа.

Исследователи MD Anderson Cancer Center впервые постарались ответить на вопрос о значении ответа на неоадъювантную ХТ, поскольку являлись первыми адептами ее в США. Самый первый ретроспективный анализ результатов лечения 150 больных с II и III стадиями рака молочной железы показал очень высокий риск локального рецидива в отсутствие адъювантной ЛТ - 27\% [11]. Среди тех больных, у которых удалось достичь полного патоморфологического ответа, процент рецидивов был ниже, но все же неприемлемо высок - 19\%. В последующей публикации Huang 2004 года эти данные были подтверждены: даже среди пациентов с достигнутым полным патоморфологическим ответом риск рецидива был высок в отсутствие адъювантного облучения (33\% против 3\% с ЛТ, $\mathrm{p}=0.006)$. При этом большинство рецидивов было зафиксировано у больных с изначальной III стадией процесса или с опухолями Т3 вне зависимости от достижения PCR [12].

В 2007 году исследователи MD Anderson обновили свой анализ, включив в него 106 больных с полным патоморфологическим ответом. Они не выявили локальных рецидивов среди больных с изначальной II стадией процесса, независимо от того, получали они адъювантную ЛТ или нет. Однако среди пациенток с III стадией эта зависимость прослеживалась очень четко: 7\% локальных рецидивов при проведении ЛТ и 33\% - без нее [13]. На основании этих данных современная тактика в MD Anderson подразумевает однозначное проведение адъювантного облучения всем больным с изначальной клинической III стадией, даже при достижении полного патоморфологического ответа.

Наконец, мы приблизились к наиболее интригующей части нашего разговора - результатам исследования, способного изменить существующую практику, исследования, выводы которого нам стоит обсуждать с пациентами, говоря о необходимости и оправданности послеоперационной ЛТ в той или иной ситуации [14]. Как было упомянуто ранее, несколько ретроспективных мультицентрических американских исследований не подразумевали проведение ЛТ после мастэктомии. Два из них - В18 и В27 - включали более 3000 больных, получивших неоадъювантную ХT с последующей органосохраняющей операцией или мастәктомией. Абсолютное большинство из них (90\%) изначально имели I или II стадии опухолевого процесса, подтвержденные методами клинической диагностики. Анализ был построен на результатах лечения больных после обоих видов оперативных вмешательств, но мы обратим внимание только на тех, что прошли через мастэктомию. Среди больных с клинически вовлеченными лимфоузлами, у которых достигнут полный патоморфологический ответ и в молочной железе, и в лимфоузлах не было отмечено локальных рецидивов, независимо от размеров первичной опухоли.

Важно отметить, что число таких больных в анализе было невелико. Наиболее высокий риск рецидива (20\% и более) отмечен среди тех пациенток, у кого, несмотря на проведенную неоадъювантную ХТ, сохранялись жизнеспособные опухолевые клетки в лимфоузлах. Именно им в первую очередь рекомендована адъювантная ЛТ. У больных с полным патоморфологическим ответом (в основном с I или II стадией на момент диагностики; мы помним, что только 10\% больных из анализа имели III стадию) риск рецидива оказался менее 5\%, и ЛТ им, скорее всего, не показана. Больные с «промежуточным (неполным) ответом», т.е. без признаков опухоли по лимфоузлам, но резидуальной опухолью в молочной железе, имели промежуточный риск локального рецидива (порядка 10\%), и таким пациенткам можно предоставить право принять собственное решение в данной ситуации.

Частота полного патоморфологического ответа неодинакова среди различных биологических подтипов рака молочной железы. Фактически, этот показатель наиболее низок среди благоприятных в плане прогноза пациенток с индолентными опухолями люминального А подтипа (7,5\%) и наиболее высок среди самых агрессивных HER-2 позитивных $(50,3 \%)$ и трижды негативных опухолей $(33,6 \%)$. Исследование Mamounas, данные которого были неоднократно представлены в прошлом году, но пока опубликованы только в виде абстракта, имело основной целью проследить показатели локального контроля в зависимости от различных факторов по результатам 12 исследований по неоадъювантной ХT [15]. Точных данных в отношении того, кто получал ЛТ после мастэктомии, а кто нет, у авторов не было, предположительно, одна треть больных прошла через адъювантную ЛТ после радикального оперативного вмешательства. Кроме того, 38\% HER-2 позитивных больных получали трастузумаб неоадъювантно и $45 \%$ - в адъюванте.

В целом, авторы обнаружили очень схожее соотношение между ответом на лечение и риском локорегионального рецидива, с наименьшим риском среди пациентов без выявленных опухолевых клеток в лимфатических узлах или молочной железе $(3,3 \%)$, и с наивысшим риском рецидива среди пациентов с остаточными опухолевыми клетками в лимфоузлах (13,1\%). Риск локального рецидива был так же оценен по биологическому подтипу. Сниженный риск рецидива был выявлен у пациентов с люминальным А подтипом опухоли (4,2\%). Самая высокая степень риска была у пациентов с HR-/Her2+ и тройной негативной опухолью (14,8\% и 12,2\%, соответственно). 
В этом анализе возраст и изначальная стадия процесса не являлись предикторами локального рецидива, и снова мы не знаем, каким образом назначалась ЛТ у этих пациенток, но наиболее вероятно, что именно пациенты с III стадией преимущественно получали ЛТ после мастәктомии.

Биологические подтипы и ответ на ХТ явились независимыми предикторами локального рецидива в мультивариантном анализе. Больные с люминальным В подтипом имели более высокий риск локального рецидива в сравнении с люминальными А опухолями, особенно при наличии вовлеченных лимфоузлов, несмотря на проведенную ХТ (13,9\% против 5,3\%). Среди HER2+ больных отрицательный рецепторный статус был ассоциирован с более высоким риском локорегионального рецидива (24,4\% при наличии резидуальной опухоли в лимфоузлах); он был также высок и у трижды негативных пациенток (до 22,1\%). Использовать эти данные рутинно в клинике пока невозможно, хотя бы по той причине, что они пока не опубликованы. Однако они дают немалую пищу для размышлений, в частности, в отношении больных с промежуточным риском локального рецидива. Так, больным с трижды негативным или HER2+ подтипом опухоли, возможно, стоит более уверенно рекомендовать адъювантную ЛТ в случае полного ответа со стороны лимфоузлов и неполной регрессии опухоли в молочной железе.

Радиационные онкологи, а особенно те, кто занимается лечением РМЖ - люди, как правило, весьма консервативные. То же самое относится и к специалистам, дебатирующим и голосующим при обсуждении текущих рекомендаций NCCN. Так, потребовалось не менее 10 лет для осознания и принятия результатов канадского и европейских исследований по гипофракционированию, чтобы убедить радиотерапевтов в приемлемости этого режима лучевой терапии после органосохраняющих операций. Скорее всего, консерваторы придадут большее значение недостаточным проспективным данным по отказу от ЛТ, нежели ретроспективному анализу В18/В27. Нынешние рекомендации утверждают, что решение о проведении ЛТ после мастэктомии должно базироваться на клинических данных до начала ХТ. Однако теперь мы знаем, что ретроспективные исследования противоречат этим рекомендациям и предполагают, что окончательная патоморфологическая оценка способна повлиять на проведение либо отказ от ЛТ.

Вот так может выглядеть алгоритм решений в отношении адъювантной ЛТ для больных с II стадией рака молочной железы после мастэктомии при отсутствии опухолевых клеток в лимфоузлах или молочной железе, с остаточной опухолью в лимфоузлах и совместное (!) решение по поводу пациенток с промежуточным риском рецидива, с учетом биологического подтипа опухоли (таблица 1).
Таблица 1. Интерпретация ретроспективных данных для возможсного практического применения в отношении больных с II стадией рака молочной железы после неоадбювантной ХТ и радикальной мастәктомии в зависимости от опухолевого ответа

\begin{tabular}{|l|c|c|c|}
\hline & Да & Возможно & Нет \\
\hline урT0 урN0 & & & $\sqrt{ }$ \\
\hline урТлюбая урN+ & $\sqrt{ } *$ & & \\
\hline урT1-3 урN0 & & $\sqrt{ } * *$ & \\
\hline
\end{tabular}

Примечание:

* Не очевидно для люминального А подтипа.

** Более очевидно для трижды негативного и Her2+ подтипов.

В настоящее время 2 рандомизированных исследования, призванных обеспечить солидный уровень доказательности комитету экспертов NCCN, производят набор больных в США. Первое набирает больных с T1-3N1 без признаков опухоли в лимфоузлах после неоадъювантной ХТ и мастэктомии для проведения ЛТ на грудную стенку и регионарные зоны или отказа от нее. Второе - Alliance - отбирает больных, не подходящих для первого исследования из-за наличия признаков жизнеспособной опухоли в лимфоузлах по данным биопсии сторожевого лимфоузла, и рандомизирует их либо в группу ЛТ, либо комбинации ее с аксиллярной лимфодиссекцией (уровни 1 и 2).

После многих лет дебатов появления результатов 3 крупных рандомизированных исследований и большого мета-анализа мы практически выяснили, какие больные нуждаются в адъювантной ЛТ после мастэктомии и сформировали рекомендации. Однако, вошедшая в широкую практику, неоадъювантная ХТ вновь спутала карты и сбила с толку врачей и пациенток. И вновь мы анализируем ретроспективные исследования и ждем новых проспективных результатов. Тем не менее, вполне может оказаться, что возможность отказа от ЛТ у пациенток при изначально пораженных лимфоузлах и клиническом ответе на ХТ может стать самым значительным, если не единственным, преимуществом неоадъвантной ХT.

\section{2. Как крупные исследования 2016 года способны повлиять на тактику ведения больных ракоп про-} статы низкого и промежсуточного риска

Согласно официальной статистике по Российской Федерации [16], с 2005 года заболеваемость раком простаты возросла практически в 4 раза: с 43,1 до 128,4 случаев на 100 тысяч населения. При этом большинство опухолей выявляется, как правило, в возрасте между 65 и 74 годами и чаще всего в стадии локализованного процесса (в 2015 году у 55,2\% больных диагностированы I и II стадии рака простаты), определяя, таким образом, своих обладателей преиму- 
щественно в группы низкого (или даже очень низкого) и промежуточного риска. Мировые исследования, произведенные на основании аутопсий, тем не менее, демонстрируют, что опухоли простаты возникают гораздо раныше и гораздо чаще, нежели демонстрирует любая статистика, и при этом мужчины в течение длительного времени не имеют ни малейшего понятия об их существовании. Примерно у 29\% мужчин в возрасте от 30 до 40 лет обнаруживаются маленькие злокачественные опухоли в простате, и этот процент возрастает до 64\% в промежутке между 60 и 70 годами. При этом лишь 1 из 38 больных раком простаты умирает по данной причине, тогда как более $2 / 3$ мужчин в возрасте за 70 продолжают жить с этой опухолью и погибают от других заболеваний.

Различия в потенциальной агрессивности этих опухолей, отчасти определяемая патоморфологами с использованием шкалы Глисон, могут являться причиной того, что часть опухолей персистирует десятилетиями, отличаясь медленным ростом, не метастазируя, никогда не обнаруживая себя симптомами и не угрожая жизни. Осознание того, что рак простаты с низкой степенью злокачественности способен вести себя столь индолентно, со временем привело к дискуссиям, не нанесет ли максимум вреда именно лечебное вмешательство в подобных ситуациях (операция, лучевая или гормональная терапия), которое однозначно спасло бы жизнь пациентам с опухолями высокой степени злокачественности. И хотя загадка, почему опухоли простаты низкой и высокой степени злокачественности столь по-разному ведут себя, до сих пор до конца не разгадана, определенные выводы в отношении тактики ведения пациентов научное сообщество все-таки сделало.

Специалисты NCCN в своих рекомендациях в отношении рака простаты не только низкого риска (опухоль Т1-T2a, Gleason $\leq 6$, уровень простатоспецифического антигена (ПСА) на момент диагностики $\leq 10$ нг/мл), но даже и промежуточного (Т2b-T2c, Gleason $\geq 7$, ПСА - 10-20 нг/мл) советуют при выборе тактики решения, прежде всего, отталкиваться от ожидаемой продолжительности жизни пациента. Если она не превышает 10 лет, то можно ограничиться только наблюдением за данным больным без принятия каких-либо решительных мер специфического воздействия на опухоль. Если ожидаемая продолжительность жизни, скорее всего, превысит 10 лет, тогда можно рассматривать и другие варианты тактики ведения пациента, среди которых, впрочем, активное наблюдение (анализ крови на ПСА не чаще одного раза в шесть месяцев при наличии клинических проявлений, а также несколько других исследований) является не просто допустимой опцией, а первым из предлагаемых экспертами вариантов для больных раком простаты низкого риска. Активное наблюдение в данных ситуациях целесообразно как минимум по трем причинам:
1) Длительная «натуральная» история рака простаты низкого риска с продолжительным бессимптомным течением, скорее всего, подразумевает такое же латентное поведение опухоли на протяжении последующих лет жизни больного;

2) В руках врача есть весьма чуткий «инструмент», позволяющий диагностировать прогрессирование процесса - анализ на ПСА, а также ряд других информативных методов исследования, в частности МРТ малого таза, и др.;

3) Сценарий активного наблюдения за подобными пациентами во избежание «перелечивания» с развитием осложнений, характерных для любого вида лечения, расценивается как вполне безопасный: не более 1\% больных низкого риска, наблюдаемых регулярно, имеют шанс умереть от рака простаты в течение 15 лет. И этот весомый аргумент был неоднократно подкреплен в течение 2016 года.

Сначала несколько слов о «перелечивании», осложнениях и роли лучевой терапии в әтом. В крупном канадском популяционном исследовании, опубликованном в начале года в журнале Cancer, авторы поставили целью тщательно оценить риск развития колоректального рака у 14164 больных раком простаты в качестве первичной опухоли, которые были пролечены за период с 1987 по 2009 годы и прослежены до момента диагностики колоректального рака или другой опухоли, смерти, эмиграции или окончания сроков исследования [17]. Эти пациенты были сопоставлены со сходными по возрасту мужчинами без подобного первичного диагноза в пропорции 1:5 (69 051 мужчин в группе сравнения). Риск колоректального рака оказался действительно выше среди больных раком простаты (HR 1,14; 95\% CI, 1,02-1,27). При этом риск развития рака прямой кишки в целом был также выше, но особенно (вдвое!) среди тех, кто был пролечен методом лучевой терапии (HR, 2,06; 95\% CI, 1,42-2,99), для которых дальнейшее регулярное обследование для исключения вторичной опухоли прямой кишки и ректо-сигмоидного отдела (колоноскопия) должно стать пожизненным. Причем данный риск даже возрастает со временем: по прошествии периода наблюдения свыше 10 лет он достигает 4,91! Это еще одна причина, почему стоит крайне взвешенно подходить к назначению лечения у больных раком простаты низкого риска, и среди всех возможных подходов лучевая терапия, особенно дистанционная, однозначно не должна стоять на первом месте.

Для сохранных больных с локализованными опухолями предстательной железы адекватно выполненная радикальная простатэктомия является методом, позволяющим избавить больного от опухоли и в большинстве случаев больше не прибегать ни к какому лечению. В рекомендациях NCCN именно радикальная простатэктомия с тазовой лимфодиссекцией или без нее обозначены в качестве метода 
выбора при раке простаты низкого и промежуточного риска, так же как и радикальная лучевая терапия в различных вариантах (дистанционная, брахитерапия либо сочетанная). До прошлого года убедительных данных 1 уровня доказательности каких-либо преимуществ того или иного из упомянутых методов над другим не существовало - пока не увидел свет крупный мета-анализ 19 исследований по данной проблеме, опубликованный в журнале European Urology в июле 2016 года опять-таки канадскими исследователями [18]. В анализ было включено огромное количество больных раком простаты низкого и промежуточного риска (118 830 пациентов) с основной целью: сравнить эффективность хирургического и лучевого лечения, опираясь на показатели общей и раково-специфической смертности. В исследованиях компонент лучевой терапии подразумевал в основном использование дистанционного облучения, реже брахитерапии изолированно либо в сочетании с дистанционным. Полученные результаты способны обескуражить специалистов в тех центрах, где лучевая терапия применяется более регулярно для лечения подобных больных, нежели простатэктомия. Риск как общей смертности (HR 1,63, 95\% confidence interval $1,54-1,73, \mathrm{p}<0,00001)$, так и раково-специфической (HR 2,08, 95\% confidence interval 1,76-2,47, p<0,00001) оказался значительно выше для пациентов, прошедших через облучение, чем для оперированных, причем анализ по подгруппам продемонстрировал ту же закономерность вне зависимости от группы риска рака простаты, режима облучения, его продолжительности и длительности периода наблюдения. Однако авторы корректно воздержались от каких-либо далеко идущих выводов (упомянув, тем не менее, что полученные данные, несомненно, должны быть обсуждены с пациентом при выборе метода лечения), поскольку на тот момент с нетерпением ожидались результаты рандомизированного исследования ProtecT, призванного поставить определенную точку во «взаимоотношениях» лучевой терапии, простатэктомии и активного наблюдения у больных раком простаты низкого и промежуточного риска.

Публикации о результатах данного исследования появились в журнале New England Journal of Medicine осенью 2016 года и затрагивали две основные темы: выживаемость и качество жизни [19, 20]. В промежутке между 1999 и 2009 годами 82429 мужчин в возрасте от 50 до 69 лет прошли скрининговое тестирование на ПСА, у 2664 из них в итоге был диагностирован локализованный рак предстательной железы, и 1643 пациентов из этой группы согласились на рандомизацию в группы активного наблюдения (545), операции (553) и лучевой терапии (545). Главной целью исследования явилась раково-специфическая смертность за средний 10-летний период наблюдения, а вторичными - риск прогрессирования, метастазирования и смертности от всех случаев. Несмотря на некоторые слабые места исследования (протокол был разработан около 20 лет назад, МРТ малого таза не была включена в базовое обследование до и после лечения, за этот период изменилась и техника облучения/оперирования, и др.), достоверность данных не вызывает сомнений благодаря рандомизации, стандартизованности лечебных подходов и скрупулезным регулярным наблюдением за пациентами. В итоге за средний период наблюдения в 10 лет раково-специфическая смертность была весьма низка независимо от примененного подхода: всего зарегистрировано лишь 17 смертных случаев, 8 из них в группе активного наблюдения, 5 - в группе хирургического лечения, и 4 - в группе радиотерапии. При этом статистически достоверной разницы не зафиксировано $(\mathrm{P}=0,48)$. В добавление к тому, различия в общей смертности между группами были также статистически не значимы $(\mathrm{P}=0,87)$. Однако активное лечебное вмешательство приводило к снижению риска прогрессирования и метастазирования по сравнению с активным наблюдением, но, в то же время, как показывают данные по качеству жизни [20], 44\% больных из группы наблюдения за весь период благополучно избежали радикального лечения и, соответственно, каких-либо осложнений. Вот в чем действительно наблюдались различия между группами, так это именно в отношении качества жизни пациентов. Так, наибольший стойкий негативный эффект на сексуальную функцию и состоятельность сфинктеров мочевыводящих путей оказало оперативное лечение, а также лучевая терапия, хотя и только в первые 6 месяцев после облучения с постепенным восстановлением. В то же время повреждение кишечника явилось эксклюзивным последствием только лучевой терапии. Все эти негативные эффекты активного лечения зеркально отразились и на общем качестве жизни. Тем не менее, в отношении частоты депрессии, обеспокоенности и общего восприятия действительности значимых отличий между группами отмечено не было.

Таким образом, 2016 год еще раз сурово подтвердил: выбор тактики ведения больных раком предстательной железы низкого риска - это компромисс, абсолютно отвергающий какую-либо авторитарность со стороны врача или пациента. Это обстоятельный, требующий времени, терпения и знаний разговор с обозначением всех возможных кратковременных и отсроченных, длительных эффектов активного лечения, определенных рисков активного наблюдения, влияния и тех и других на качество жизни, заканчивающийся в итоге выбором наиболее подходящего подхода, основанного на полной информированности больного.

\section{6: шаги к прогрессу}

1. Как комбинация таргетной/иммунотерапии и лучевой терапии может изменить подход кле- 
чению радиорезистентных опухолей различных локализаций. Абскопальный эфбект облучения

За последнее десятилетие появилось множество новых данных о драйверных мутациях, что привело к беспрецедентным успехам в лечении распространенных опухолей. Мы видим значительное улучшение показателей выживаемости, в частности, у больных со специфическими для немелкоклеточного рака легкого (НМКРЛ) или меланомы мутациями, на которые можно действовать направленно. Проблема состоит в том, как наиболее оптимально состыковать таргетную терапию с локальным лечением, в частности, с лучевой терапией, в тех ситуациях, когда речь об оперативном лечении в силу распространенности процесса не идет, но при этом без локального воздействия лечение представляется неполноценным. Первый вопрос, который мы должны задать себе - а какова же природа кооперации между таргетными агентами и излучением?

Наиболее частая и логичная цель комбинирования системных химиотерапевтических агентов с облучением - радиосенсибилизация за счет усиления поражаемости опухолевых клеток цитотоксическими препаратами, причем механизмы взаимодействия могут быть разнообразны: ингибирование ферментов репарации ДНК вкупе с непосредственным повреждающим воздействием на молекулу ДНК, влияние на процессы реоксигенации клеток, синхронизация клеточного цикла и др. Однако есть и другие механизмы, через которые излучение и лекарственные препараты (в том числе и таргетные) могут совместно потенциировать клеточную гибель. Одна из таких моделей названа пространственной кооперацией. Суть ее в том, что ионизирующее излучение фокусируется на первичной опухоли, а лекарственный агент воздействует на отдаленные опухолевые клетки или очаги. В данном случае неважно, проводится ли лечение с использованием этих двух методов одновременно или последовательно. Существуеттакже биологическая кооперация: есть подгруппа опухолевых клеток, в большей степени чувствительных к действию излучения, и подгруппа клеток, чувствительных только к лекарственным препаратам, и, снова, вне зависимости от последовательности. Наконец, существует временная (или преходящая) кооперация. В данной модели излучение сенсибилизирует опухолевые клетки к последующей лекарственной терапии, при этом частично достигая цели, а дальнейшее введение лекарственного агента позволяет достичь полной стерилизации - либо наоборот. Это и есть основная задача одновременного химиолучевого лечения.

В отношении НМКРЛ практически все из известных таргетных препаратов, применяемых в лечении таких больных, к настоящему моменту были протестированы в комбинации с облучением, как минимум, в доклинических или клинических исследованиях I фазы. Наибольшее количество работ посвящено ин- гибиторам ангиогенеза и рецепторов эпидермального фактора роста: есть даже несколько исследований III фазы. Еще на рубеже веков в исследования in vitro и in vivo было обнаружено инверсное взаимоотношение между гиперэкспрессией дикого типа EGFR и опухолевым ответом на облучение [21, 22], и сигнальный путь рецептора EGFR был описан как путь классической радиорезистентности через целый ряд механизмов [23]. Следовательно, ингибирование рецепторов EGFR перекрывает сигнальные пути радиорезистентности и способствует увеличению противоопухолевой активности излучения через угнетение клеточного цикла, индукцию апоптоза и действие на ускоренно репопулирующие клетки. При этом клеточные линии НМКРЛ с соматической активирующей мутацией представляют собой высокочувствительный к облучению фенотип опухоли, и хотя в настоящее время данных о точном механизме взаимодействия излучения и ингибиторов тирозинкиназы при EGFR-мутантном подтипе крайне мало, ряд исследователей поддерживает версию аддитивного, нежели синергетического эффекта.

Однако большинство опубликованных исследований с различными представителями группы анти-EGFR в комбинации с облучением продемонстрировали результаты, как минимум, сопоставимые с традиционными режимами химиотерапии.

Несмотря на теоретическую логику (перестройка микрососудистого русла с последующей реоксигенацией опухоли, усиленный клеточный апоптоз вследствие индуцированных повреждений клеток эндотелия и др.), до сих пор комбинация лучевой терапии и ингибиторов ангиогенеза являла собой пример терапевтического фиаско в большинстве случаев. Бевацизумаб в исследовании II фазы по химиолучевому лечению продемонстрировал выраженную токсичность у большого числа больных, вплоть до образования трахеобронхиальных свищей [24]. Другие представители этой группы также не показали какихлибо преимуществ в комбинации с облучением. Но в любом случае, комбинация химиолучевого лечения с новыми терапевтическими агентами представляется весьма привлекательной терапевтической опцией для больных местнораспространенным НМКРЛ, а посему немалое количество исследований проводится в настоящее время.

Для рака пищевода таргетная терапия, как принято считать, и подавно находится лишь в начале большого пути, и до первых позитивных результатов пока еще далеко. В применении к плоскоклеточному раку и гастроэзофагеальной аденокарциноме пищевода к настоящему моменту описано и изучено несколько потенциальных опухолевых маркеров и мишеней, прежде всего имеющих отношение к регуляции роста (EGFR, HER2/neu, Ki-67), ангиогенезу (VEGF), воспалению (COX-2), контролю клеточной пролиферации (p16, p21, cyclinD1), апоптозу (p53, bax, bcl-2) и др. 
Большинство из них изучались изолированно, как предикторы клинической эффективности, в частности, ответа на предоперационную химиотерапию и химиолучевое лечение.

Повышенная экспрессия EGFR, согласно иммуногистохимическим исследованиям, весьма часто наблюдается при раке пищевода, почти у $80 \%$ больных, при этом амплификация гена EGFR встречается, по данным разных авторов, в 8-30\% случаев при гастроэзофагеальной аденокарциноме. Многочисленные исследования показали, что увеличенная экспрессия EGFR ассоциирована со статистически значимым снижением общей выживаемости больных раком пищевода. На основании этих выводов, в мире было инициировано большое количество исследований I-II фазы по применению малых молекул ингибиторов тирозинкиназы и моноклональных антител в лечении больных гастроэзофагеальным раком. Анализ, проведенный на основании биопсий, показал отсутствие корреляции между экспрессией EGFR и противоопухолевой активностью таргетных препаратов, а также их ограниченную активность у больных с плоскоклеточным раком при абсолютном отсутствии эффекта при аденокарциноме пищевода. Исследование III фазы RTOG 0436 (2014 год) по добавлению цетуксимаба к стандартной комбинации цисплатина и паклитаксела на фоне лучевой терапии не выявило преимуществ в плане клинического ответа или общей выживаемости [25]. Есть еще и другие исследования II-III фазы, продемонстрировавшие ухудшение общей выживаемости и более высокую токсичность с цетуксимабом, в результате чего препарат не рекомендован к применению для химиолучевого лечения рака пищевода. Видимо, исследователям еще только предстоит найти верное направление в практическом применении таргетных препаратов с лучевой терапией, коли теоретический базис подразумевает определенный успех данной комбинации.

Однако уже есть и весьма яркие и удачные случаи, к примеру, результаты ретроспективного исследования, выполненного в университете Колорадо [26]. В соответствии с практикой данного центра, больные с экстракраниальными олигометастазами ALK-позитивного НМКРЛ получали кризотиниб и затем стереотаксическую лучевую терапию при прогрессировании с целью локальной абляции. При вполне толерантной токсичности лечения одногодичный локальный контроль в очагах, не ответивших на лечение кризотинибом и облученных однократно в дозе $\geq 25$ Гр либо <25 Гр, составил $100 \%$ и 60\%, соответственно. Исследователи обнаружили, что стабилизация после облучения позволяет пролонгировать лечение кризотинибом, что коррелировало с улучшенными показателями общей выживаемости (2-летняя выживаемость при нахождении на кризотинибе свыше 12 месяцев либо менее 12 месяцев составила $72 \%$ и $12 \%$, соответственно). Основная идея состояла в том, чтобы поддерживать в стабильном состоянии олигометастатический процесс без дальнейшего прогрессирования. Это как раз и есть великолепный пример биологической кооперации: в одном теле пациента присутствуют гетерогенные опухолевые очаги, и те из них, которые проявили резистентность к кризотинибу, подвергаются успешному воздействию лучевой терапией.

Еще один пример теоретически обоснованной комбинации интенсивного облучения с таргетной терапией при НМКРЛ с метастазами в головной мозг. Облучение головного мозга приводит к разрушению гематоэнцефалического барьера за счет двухволновой гибели эндотелиальных клеток: раннего апоптоза (в течение ближайших 24 часов после воздействия ионизирующим излучением) и отсроченной митотической гибели вследствие повреждений молекулы ДНК (от 72 часов и на протяжении почти месяца после облучения). Подобные эффекты наблюдались при проведении лучевой терапии как на весь объем головного мозга (при минимальных дозах 20-30 Гр за 10-15 фракций), так и локального облучения метастатических очагов (как показывают доклинические исследования,доза в 10 Гр за фракцию и выше при фокусном стереотаксическом облучениитакже способствует разрушению барьера). Логично предположить, что после облучения головного мозга проникающая способность лекарственных препаратов должна быть выше, хотя пока эти данные весьма противоречивы. Вполне возможно, что проникновение малых молекул ингибиторов тирозинкиназы окажется практически беспрепятственным, и комбинация облучения с ингибиторами EGFR будет являть собой пример преодоления вышеупомянутой радиорезистентности опухоли. Недавний совокупный анализ результатов 8 проспективных исследований, включающих 980 больных НМКРЛ с метастазами в головной мозг, получавших облучение на фоне эрлотиниба или гефитиниба, продемонстрировал значимое преимущество добавления этих препаратов в лечебную схему в плане объективного ответа $(\mathrm{HR}=1,56, \mathrm{P}=0,0008)$, пролонгированного периода до интракраниального прогрессирования ( $\mathrm{HR}=0,58$, $\mathrm{P}=0,03)$ и медианы выживаемости $(\mathrm{HR}=0,68, \mathrm{P}=0,04)$, хотя оценка результатов в соответствии с EGFR статусом авторами не предоставлялась [27].

В настоящее время инициировано и продолжает набор пациентов большое количество проспективных исследований по совместному применению таргетных препаратов различных групп, в том числе PARP и ALK ингибиторов, с лучевой терапией при местнораспространенном и метастатическом НМКРЛ, но период ожидания их результатов остается пока немалым. Видимо, пройдет еще много времени, прежде чем мы получим конкретные рекомендации в отношении оптимальных комбинаций, доз и временного 
соотношения таргетных препаратов и ионизирующей радиации.

Возникает еще один весьма провокационный вопрос: способна ли лучевая терапия обеспечить нечто большее, чем просто локальное воздействие с целью влияния на локальный контроль? Что мы в действительности знаем о системных эффектах излучения? В исследовании из университета Чикаго [28] мыши с меланомой В16 подвергались однократному облучению в дозе 20 Гр; подобное воздействие даже на столь агрессивную радиорезистентную опухоль способно приводить к значительной регрессии. Однако авторы работали с диким типом мышей с обедненным составом Т-лимфоцитов. Нет ничего удивительного в том, что когда мы облучаем опухоль, то со временем получаем опухолевый ответ. Интереснее тот факт, что когда эксперимент проводится на животном с угнетенной иммунной системой, опухоль продолжает расти, даже при увеличении дозы облучения. Повторяя эксперименты, исследователи вновь и вновь убеждались, что в данной ситуации возможность контролировать опухолевый процесс с помощью излучения утрачивалась. Подобные исследования известны науке еще с конца 70-х годов прошлого века: Stone и соавторы, работая с животными с индуцированными фибросаркомами, определили дозу лучевой терапии, позволяющую контролировать более 50\% опухолей [29]. Однако при стимулировании иммунной системы животного неочищенными бактериальными взвесями они отметили, что требуются гораздо меньшие дозы облучения для достижения такого же контроля и, наоборот, при введении животных в иммуносупрессию даже значительное повышение доз лучевой терапии не всегда способствовало контролю над опухолью. Видимо, существует некое взаимодействие с иммунной системой, что открывает новые горизонты для комбинации иммунопрепаратов и локального облучения и позволяет механистически представить себе то, что мы называем «абскопальный әффект» (эффект, проявляющийся не в месте облучения).

Известен яркий пример абскопального эффекта, клинический случай пожилой пациентки, которая получила химиолучевое лечение с последующей стереотаксической лучевой терапией (СЛТ) на два различных первичных очага аденокарциномы легких. До облучения у нее были обнаружены метастатические очаги в надпочечнике и плечевой кости, которые спонтанно регрессировали после СЛТ. Подобные типы ответа редки, но позволяют поддерживать теорию, что лучевая терапия, помимо ее прямого воздействия на молекулу ДНК, продуцирует иммуногенную клеточную гибель. То есть, по мере гибели опухолевых клеток высвобождается огромное количество опухолевых антигенов, которые стимулируют специфичный иммунный противоопухолевый ответ не только в области облученного очага, но и в отдалении, причем этот эффект однозначно более выражен при использовании аблативных доз лучевой терапии крупными фракциями, нежели при конвенциональном облучении [30].

Мы вступаем в эру иммунотерапии, эру широкого применения ингибиторов контрольных точек, активность которых однозначно не ограничивается только меланомой или НМКРЛ, известным большим количеством мутаций. Можем ли мы в перспективе комбинировать лучевую терапию с чекпойнт ингибиторами для улучшения показателей ответа на лечение, и какова в данном случае была бы модель взаимодействия? Потенциально, высокие дозы излучения способны стимулировать массивную клеточную гибель. Высвобождающиеся опухолевые антигены захватываются впоследствии детерминированными клетками с предоставлением антигенной информации специфичным Т-лимфоцитам, осуществляющим захват опухолевых клеток; они впоследствии способны не только воздействовать на первичную опухоль, но и на любые очаги диссеминации, поскольку циркулируют по всему организму. Ингибиторы контрольных точек действуют именно в данной точке приложения: при наличии опухолеспецифического захвата Т-лимфоцитами предотвращается нарушение регуляции Т-клеточного ответа. Модель кооперации основана на этом же. В отсутствии антигенов (если опухоль недостаточно иммуногенна) чекпойнт ингибиторы не работают. Однако если мы используем лучевую терапию для стимуляции увеличения количества антигенов и добиваемся иммуногенной клеточной гибели в опухолевом очаге, мы можем стимулировать активность Т-лимфоцитов и, следовательно, повысить эффективность ингибиторов контрольных точек. Немалое количество работ по данной проблеме опубликовано в течение последних пяти лет, в основном доклинических или исследований первой фазы, и это еще одно подтверждение огромного интереса к комбинации лучевой и иммунотерапии.

Иммунотерапия успела зарекомендовать себя в качестве четвертого оплота специфического противоопухолевого лечения. В настоящее время основное внимание будет уделяться предиктивным и прогностическим факторам ответа на терапию, пониманию механизма взаимодействия иммунотерапии и облучения и решению, как наилучшим образом внедрить иммунотерапию в радикальное и паллиативное лечение опухолей разных локализаций. Мы вступаем в новую эру, проходя через алгоритмы, основанные на стадировании, к решениям, базирующимся на биологии различных опухолей. Оптимальная стратегия все еще не определена, но тем интереснее представляется будущее для исследователя.

2. 2016: о новой ролилучевой терапии влечении «традиционно» радиорезистентных опухолей

В завершение речь пойдет о возможностях лучевой терапии в лечении онкологических больных, которые 
в подавляющем большинстве на момент диагностики признаются инкурабельными по причине невозможности оперативного лечения и неэффективности (как принято считать) других методов специфического воздействия. Гепатоцеллюлярная карцинома и внутрипеченочная холангиокарцинома - не часто встречающиеся опухоли из разряда первичных поражений печени, но крайне плохо поддающиеся лечению. Хирургический метод считается для них основным и чуть ли не единственно эффективным, однако, лишь 30\% больных на момент диагностики признаются потенциально операбельными. Остальным 70\% достаются, в лучшем случае, паллиативные методы локального воздействия (радиочастотная абляция) или паллиативная химиотерапия гемцитабином и цисплатином и не более 7-12 месяцев средней выживаемости. Лучевая терапия в рекомендациях традиционно упоминается одной строчкой под грифом «возможно» без конкретизации, ибо желающих заниматься столь неблагодарным делом - облучением печени - всегда было мало. Но, тем не менее, энтузиасты из числа радиотерапевтов облучали подобных пациентов на протяжении десятилетий. В 2016 году в Journal of Clinical Oncology были опубликованы результаты нескольких исследований по данной проблеме. Одно из них, вышедшее из MD Anderson Cancer Center, представляет собой ретроспективный анализ результатов 3-D конформного облучения 79 больных с верифицированными внутрипеченочными опухолями со средним диаметром 7,9 см (от 2,2 до 17 см) за период с 2002 по 2014 годы [31]. Различные варианты фракционирования были применены исследователями в зависимости от облучаемого объема, и в пересчете на биологически эквивалентные дозы (БЭД) подавляющее число больных с крупными опухолями получили БЭД 800,5 Гр, и 19 пациентов - более 80,5 Гр. Несмотря на то, что почти половина (48\%) больных прогрессировали после лечения, исследователям удалось достичь показателей средней выживаемости и выживаемости без прогрессирования в 30 месяцев! При этом только подведенная доза лучевой терапии оказалась единственным предиктором для общей выживаемости и локального контроля. Больные с подведенной к очагу БЭД свыше 80,5 Гр (что возможно при использовании укороченных гипофракционированных режимов) имели 3-летний локальный контроль 78\% против 45\% тех, у кого БЭД были меньше; медиана выживаемости у интенсивно облученных больных не была достигнута за исследуемый период по сравнению с 27 месяцами из группы БЭД менее 80,5 Гр. Токсичность лечения оказалась вполне приемлемой и, что интересно, получение больными химиотерапии, как на этапе до облучения, так и после, не оказало влияния на общую выживаемость.

Исследователи из университета Мичиган сравнили свои результаты стереотаксического облучения неоперабельных очагов неметастатической гепатоцеллюлярной аденокарциномы (63 больных) с более традиционным паллиативным воздействием - радиочастотной абляцией (РЧА, 161 больной) и выяснили, что процент больных без локального прогрессирования в течение 1 и 2 лет оказался выше в группе стереотаксической лучевой терапии по сравнению с РЧА (97,4\% и 83,8\% против 83,6\% и 80, 2\%, соответственно). При этом успех лечения с РЧА зависел от размеров опухоли, тогда как для лучевой терапии размер особой роли не играл: для очагов более 2 см в диаметре период без локального прогрессирования был ниже для РЧА (HR, 3,35; P =0,025). Ранние осложнения лечения 3 степени и выше наблюдались у 11 больных после РЧА и в два раза реже при облучении [32].

Наконец, появились и проспективные результаты гипофракционированного облучения больных с гепатоцеллюлярной карциномой или внутрипеченочной холангиокарциномой - мультицентрическое исследование II фазы, с унифицированной методикой облучения и оценки результатов [33]. 83 пациента с опухолями размером 5 см в среднем (от 2 см до 12 см) получили гипофракционированное протонное облучение в средней дозе 58 Гр за 15 фракций, если очаги располагались в пределах 2 см от ворот печени, и 67,5 Гр за 15 фракций, если дальше 2 см. 2 -летний локальный контроль составил 94,8\% для первичных аденокарцином печени и 94,1\% - для внутрипеченочных холангиокарцином, а 2-летняя выживаемость - 63,2\% и 46,5\%, соответственно. Похоже, лучевая терапия может стать достойной и разумной альтернативой хирургическому лечению. Осталось дождаться результатов исследований III фазы - и переломить отношение практикующих врачей, прежде всего, хирургов и радиотерапевтов, к данной патологии для того, чтобы хотя бы у части из подобных больных появился шанс на более долгую жизнь в стабильном состоянии.

\section{Список литературы}

1. Overgaard M., Hansen P.S., Overgaard J.C, Andersson M., Bach F., Kjaer M., et al., for the Danish Breast Cancer Cooperative Group 82b Trial. Postoperative Radiotherapy in High-Risk Premenopausal Women with Breast Cancer Who Receive Adjuvant Chemotherapy. N Engl J Med 1997; 337: 949-955.

2. Overgaard M., Jensen M.B., Overgaard J., Hansen P.S., Rose C., Andersson M., et al. Postoperative radiotherapy in high-risk postmenopausal breast-cancer patients given adjuvant tamoxifen: Danish Breast Cancer Cooperative Group DBCG 82c randomized trial. Lancet. 1999; 353(9165): 1641-8. 
3. Ragaz J., Olivotto I.A., Spinelli J.J., Phillips N., Jackson S.M., Wilson K.S., et al. Locoregional radiation therapy in patients with high-risk breast cancer receiving adjuvant chemotherapy: 20-year results of the British Columbia randomized trial. J Natl Cancer Inst. 2005; 97(2): 116-26.

4. Overgaard M., Nielsen H.M., Overgaard J. Is the benefit of postmastectomy irradiation limited to patients with four or more positive nodes, as recommended in international consensus reports? A subgroup analysis of the DBCG 82 b\&c randomized trials. RadiotherOncol. 2007; 82: 247-53.

5. Clarke M., Collins R., Darby S., Davies C., Elphinstone P., Evans V., et al. Effects of radiotherapy and of differences in the extent of surgery for early breast cancer on local recurrence and 15-year survival: an overview of the randomized trials. Lancet 2005; 366(9503): 2087-106.

6. EBCTCG (Early Breast Cancer Trialists' Collaborative Group). Effect of radiotherapy after mastectomy and axillary surgery on 10-year recurrence and 20-year breast cancer mortality: meta-analysis of individual patient data for 8135 women in 22 randomized trials. Lancet 2014; 383: 2127-35.

7. Taghian A., Jeong J.-H., Mamounas E., Anderson S., Bryan J., Deutsch M., Wolmark N. Patterns of locoregional failure in patients with operable breast cancer treated by mastectomy and adjuvant chemotherapy with or without tamoxifen and without radiotherapy: results from five national surgical adjuvant breast and bowel project randomized clinical trials. J ClinOncol 2004; 22: 4247-4254.

8. Taghian A.G., Jeong J.J., Mamounas E.P., Parda D.S., Deutsch M., Costantino J.P., Wolmark N. Low locoregional recurrence rate among node-negative breast cancer patients with tumors $5 \mathrm{~cm}$ or larger treated by mastectomy, with or without adjuvant systemic therapy and without radiotherapy: results from five national surgical adjuvant breast and bowel project randomized clinical trials. J ClinOncol 2006; 24: 3927-3932.

9. Recht A., Comen E.A., Fine R.E., et al. Postmastectomy radiotherapy: an American Society of Clinical Oncology, American Society for Radiation Oncology, and Society of Surgical Oncology focused guideline update. J Clin Oncol2016; 34: 4431-4442.

10. Rastogi P., Anderson S.J., Bear H.D., et al. Preoperative chemotherapy: updates of National Surgical Adjuvant Breast and Bowel Project protocols B-18 and B-27. J ClinOncol 26: 778-785.

11. Buchbolz T.A., Tucker S.L., Masullo L., et al. Predictors of local-regional recurrence after neoadjuvant chemotherapy and mastectomy without radiation. J ClinOncol 2002; 20(1): 17-23.

12. Huang E.H., Tucker S.L., Strom E.A., et al. Postmastectomy radiation improves local-regional control and survival for selected patients with locally advanced breast cancer treated with neoadjuvant chemotherapy and mastectomy. J ClinOncol 2004; 22: 4691-4699.

13. McGuire S.E., Gonzalez-Angulo A.M., Huang E.H. et al. Postmastectomy radiation improves the outcome of patients with locally advanced breast cancer who achieves a pathologic complete response to neoadjuvant chemotherapy. Int J RadiatOncolBiol Phys. 2007; 68(4): 1004-9.

14. Mamounas E.P., Anderson S.J., Dignam J.J., et al. Predictors of locoregional recurrence after neoadjuvant chemotherapy: results from combined analysis of National Surgical Adjuvant Breast and Bowel Project B-18 and B-27. J ClinOncol 2015; 30: 3960-3966.

15. Mamounas E.P., Cortazar P., Zhang L., et al. Locoregional recurrence (LRR) after neoadjuvant chemotherapy (NAC): Pooled-analysis results from the collaborative trials in neoadjuvant breast cancer (CTNeoBC). J ClinOncol 32, 2014 (suppl 26; abstr 61).

16. Под ред. Каприна А.Д., Старинского В.В., Петровой Г.В. Состояние онкологической помощи населению России в 2015 году. М.: МНИОИ им. П.А. Герцена, филиал ФГБУ «НМИРЦ» Минздрава России, 2016,илл. - 236 с.

17. Desautels D., Czaykowski P., Nugent Z., Demers A.A., Mabmud S.M., Singh H. Risk of colorectal cancer after the diagnosis of prostate cancer: A population-based study. Cancer 2016, 15; 122(8): 1254-60.

18. Wallis C.J., Saskin R., Choo R., Herschorn S., Kodama R.T., Satkunasivam R., Shab P.S., Danjoux C., Nam R.K. Surgery versus radiotherapy for clinically-localized prostate cancer: a systematic review and meta-analysis. Eur Urol. 2016; 70(1): 21-30.

19. Hamdy F.C., Donovan J.L., Lane J.A., Mason M., Metcalfe C., Holding P., Davis M., Peters T.J., Turner E.L., Martin R.M., OxleyJ., Robinson M., Staffurth J., Walsh E., Bollina P., CattoJ., Doble A., Doherty A., Gillatt D., Kockelbergh R., Kynaston H., Paul A., Powell P., Prescott S., Rosario D.J., Rowe E., Neal D.E.; ProtecT Study Group. 10-Year outcomes after monitoring, surgery, or radiotherapy for localized prostate cancer. N Engl J Med. 2016 Oct 13; 375(15): 1415-1424.

20.Donovan J.L., Hamdy F.C., Lane J.A., Mason M., Metcalfe C., Walsh E., Blazeby J.M., Peters T.J., Holding P., Bonnington S., Lennon T., Bradshaw L., Cooper D., Herbert P., Howson J., Jones A., Lyons N., Salter E., Thompson P., Tidball S., Blaikie J., Gray C., Bollina P., Catto J., Doble A., Doherty A., Gillatt D., Kockelbergh R., Kynaston H., Paul A., Powell P., Prescott S., Rosario D.J., Rowe E., Davis M., Turner E.L., Martin R.M., Neal D.E.; ProtecT Study Group. Patient-reported outcomes after monitoring, surgery, or radiotherapy for prostate cancer. N Engl J Med. 2016 Oct 13; 375(15): 1425-1437.

21. Liang K., Ang K.K., Milas L., Hunter N., Fan Z. The epidermal growth factor receptor mediates radioresistance. Int J RadiatOncolBiol Phys. 2003; 57: 246-254.

22. Akimoto T., Hunter N.R., Buchmiller L., Mason K., Ang K.K., Milas L. Inverse relationship between epidermal growth factor receptor expression and radiocurability of murine carcinomas. Clin Cancer Res. 1999; 5: 2884-2890.

23. Khalifa J., Amini A., Popat S., Gaspar L.E., Faivre-Finn C. Brain metastases from NSCLC: radiation therapy in the era of targeted therapies. Journal of Thoracic Oncology 2016; 11(10): 1627-1643. 
24. Spigel D.R., HainsworthJ.D., Yardley D.A., et al. Tracheoesophageal fistula formation in patients with lung cancer treated with chemoradiation and bevacizumab. J ClinOncol 2010; 28: 43-8.

25. Kachnic L.A., Moughan J., Suntharalingam M., Ilson D.H., Konski A.A., Burrows W., Anker C., Bar Ad V., Thakrar H.V., Hayes J.P., Gore E.M., Kavadi V.S., Komaki R.U., Raben A., Giguere J.K., Pollock J., Greenberger J.S., Videtic G.M., Roof K.S., Watkins Bruner D. Patient-reported outcomes (PROs) in NRG Oncology/RTOG 0436: a Phase 3 trial evaluating the addition of cetuximab to paclitaxel, cisplatin, and radiation for esophageal cancer treated without surgery. Int J RadiatOncolBiol Phys 2016, 96(2): S31.

26. Gan G.N., Weickhardt A.J., Scheier B., Doebele R.C., Gaspar L.E., Kavanagh B.D., Camidge D.R. Stereotactic radiation therapy can safely and durably control sites of extra-central nervous system oligoprogressive disease in anaplastic lymphoma kinase-positive lung cancer patients receiving crizotinib. Int J RadiatOncolBiol Phys. 2014 Mar 15; 88(4): 892-8.

27. Luo S., Chen L., Chen X., Xie X. Evaluation on efficacy and safety of tyrosine kinase inhibitors plus radiotherapy in NSCLC patients with brain metastases. Oncotarget. 2015; 6: 16725-16734.

28. Lee Y., Aub S.L., Wang Y., Burnette B., Wang Y., Meng Y., Beckett M., Sharma R., Chin R., Tu T., Weichselbaum R.R., $F u$ Y.X. Therapeutic effects of ablative radiation on local tumor require CD8+ T cells: changing strategies for cancer treatment. Blood. 2009 Jul 16; 114(3): 589-95.

29. Stone H.B., Peters L.J., Milas L. Eff ect of host immune capability on radiocurability and subsequent transplantability of a murine fi brosarcoma. J Natl Cancer Inst 1979; 63: 1229-35.

30. Siva S., Callahan J., MacManus M.P., Martin O., Hicks R.J., Ball D.L. Abscopal effects after conventional and stereotactic lung irradiation of non-small-cell lung cancer. Journal of Thoracic Oncology 2013; 8(8): e71-72.

31. Tao R., Krishnan S., Bhosale P.R., Javle M.M., Aloia T.A., ShroffR.T., Kaseb A.O., Bishop A.J., Swanick C.W., Koay E.J., Thames H.D., Hong T.S., Das P., Crane C.H. Ablative radiotherapy doses lead to a substantial prolongation of survival in patients with inoperable intrahepatic cholangiocarcinoma: a retrospective dose response analysis. J ClinOncol 2016; 34: 219-226.

32. Wahl D.R., Stenmark M.H., Tao Y., Pollom E.L., Caoili E.M., Lawrence T.S., Schipper M.J., Feng M. Outcomes after stereotactic body radiotherapy or radiofrequency ablation for hepatocellular carcinoma. J ClinOncol. 2016; 34(5): 452-9.

33. Hong T.S., WoJ.Y., Yeap B.Y., Ben-Josef E., McDonnell E.I., Blaszkowsky L.S., Kwak E.L., Allen J.N., ClarkJ.W., Goyal L., Murphy J.E., Javle M.M., WolfgangJ.A., Drapek L.C., Arellano R.S., Mamon H.J., Mullen J.T., Yoon S.S., Tanabe K.K., Ferrone C.R., Ryan D.P., DeLaney T.F., Crane C.H., ZbuA.X. Multi-institutional phase ii study of high-dose hypofractionated proton beam therapy in patients with localized, unresectable hepatocellular carcinoma and intrahepatic cholangiocarcinoma. J ClinOncol 2016; 34: 460-468.

\section{References}

1. Overgaard M., Hansen P.S., Overgaard J.C, Andersson M., Bach F., Kjaer M., et al., for the Danish Breast Cancer Cooperative Group 82b Trial. Postoperative Radiotherapy in High-Risk Premenopausal Women with Breast Cancer Who Receive Adjuvant Chemotherapy. N Engl J Med 1997; 337: 949-955.

2. Overgaard M., Jensen M.B., Overgaard J., Hansen P.S., Rose C., Andersson M., et al. Postoperative radiotherapy in high-risk postmenopausal breast-cancer patients given adjuvant tamoxifen: Danish Breast Cancer Cooperative Group DBCG 82c randomized trial. Lancet. 1999; 353(9165): 1641-8. doi: 10.1016/S0140-6736(98)09201-0. PMID: 10335782.

3. Ragaz J., Olivotto I.A., Spinelli J.J., Phillips N., Jackson S.M., Wilson K.S., et al. Locoregional radiation therapy in patients with high-risk breast cancer receiving adjuvant chemotherapy: 20 -year results of the British Columbia randomized trial. J Natl Cancer Inst. 2005; 97(2): 116-26. doi: 10.1093/jnci/djh297. PMID: 15657341.

4. Overgaard M., Nielsen H.M., Overgaard J. Is the benefit of postmastectomy irradiation limited to patients with four or more positive nodes, as recommended in international consensus reports? A subgroup analysis of the DBCG 82 b\&c randomized trials. RadiotherOncol. 2007; 82: 247-53.

5. Clarke M., Collins R., Darby S., Davies C., Elphinstone P., Evans V., et al. Effects of radiotherapy and of differences in the extent of surgery for early breast cancer on local recurrence and 15-year survival: an overview of the randomized trials. Lancet 2005; 366(9503): 2087-106. doi: 10.1016/S0140-6736(05)67887-7. PMID: 16360786.

6. EBCTCG (Early Breast Cancer Trialists' Collaborative Group). Effect of radiotherapy after mastectomy and axillary surgery on 10 -year recurrence and 20 -year breast cancer mortality: meta-analysis of individual patient data for 8135 women in 22 randomized trials. Lancet 2014; 383: 2127-35.

7. Taghian A., Jeong J.-H., Mamounas E., Anderson S., Bryan J., Deutsch M., Wolmark N. Patterns of locoregional failure in patients with operable breast cancer treated by mastectomy and adjuvant chemotherapy with or without tamoxifen and without radiotherapy: results from five national surgical adjuvant breast and bowel project randomized clinical trials. J ClinOncol 2004; 22: 4247-4254. doi: 10.1200/JCO.2004.01.042. PMID: 15452182.

8. Taghian A.G., Jeong J.J., Mamounas E.P., Parda D.S., Deutsch M., Costantino J.P., Wolmark N. Low locoregional recurrence rate among node-negative breast cancer patients with tumors $5 \mathrm{~cm}$ or larger treated by mastectomy, with or without adjuvant systemic therapy and without radiotherapy: results from five national surgical adjuvant breast and bowel project randomized clinical trials. J ClinOncol 2006; 24: 3927-3932. 
9. Recht A., Comen E.A., Fine R.E., et al. Postmastectomy radiotherapy: an American Society of Clinical Oncology, American Society for Radiation Oncology, and Society of Surgical Oncology focused guideline update. J ClinOncol2016; 34: 4431-4442.

10. Rastogi P., Anderson S.J., Bear H.D., et al. Preoperative chemotherapy: updates of National Surgical Adjuvant Breast and Bowel Project protocols B-18 and B-27. J ClinOncol 26: 778-785. doi: 10.1200/JCO.2007.15.0235. PMID: 18258986.

11.BuchbolzT.A., TuckerS.L., Masullo L., et al. Predictors of local-regional recurrence after neoadjuvant chemotherapy and mastectomy without radiation. J ClinOncol 2002; 20(1): 17-23.

12. Huang E.H., Tucker S.L., Strom E.A., et al. Postmastectomy radiation improves local-regional control and survival for selected patients with locally advanced breast cancer treated with neoadjuvant chemotherapy and mastectomy. J ClinOncol 2004; 22: 4691-4699. doi: 10.1200/JCO.2004.11.129. PMID: 15570071.

13. McGuire S.E., Gonzalez-Angulo A.M., Huang E.H. et al. Postmastectomy radiation improves the outcome of patients with locally advanced breast cancer who achieves a pathologic complete response to neoadjuvant chemotherapy. Int J RadiatOncolBiol Phys. 2007; 68(4): 1004-9.

14. Mamounas E.P., Anderson S.J., Dignam J.J., et al. Predictors of locoregional recurrence after neoadjuvant chemotherapy: results from combined analysis of National Surgical Adjuvant Breast and Bowel Project B-18 and B-27. J ClinOncol 2015; 30: 3960-3966. doi: 10.1200/JCO.2011.40.8369. Epub 2012 Oct 1. PMID: 23032615.

15. Mamounas E.P., Cortazar P., Zhang L., et al. Locoregional recurrence (LRR) after neoadjuvant chemotherapy (NAC): Pooled-analysis results from the collaborative trials in neoadjuvant breast cancer (CTNeoBC). J ClinOncol 32, 2014 (suppl 26; abstr 61).

16. Kaprin A.D., Starinskyi V.V., Petrova G.V. (ed.). Status of cancer care of Russian population in 2015. M., Moscow Research Institute of Oncology named after Herzen, Clinic. 2016: 236.

17. Desautels D., Czaykowski P., Nugent Z., Demers A.A., Mabmud S.M., Singh H. Risk of colorectal cancer after the diagnosis of prostate cancer: A population-based study. Cancer 2016, 15; 122(8): 1254-60. doi: 10.1002/cncr.29919. Epub 2016 Feb 25. PMID: 26919270.

18. Wallis C.J., Saskin R., Choo R., Herschorn S., Kodama R.T., Satkunasivam R., Shab P.S., Danjoux C., Nam R.K. Surgery versus radiotherapy for clinically-localized prostate cancer: a systematic review and meta-analysis. Eur Urol. 2016; 70(1): 21-30.

19. Hamdy F.C., Donovan J.L., Lane J.A., Mason M., Metcalfe C., Holding P., Davis M., Peters T.J., Turner E.L., Martin R.M., Oxley J., Robinson M., Staffurth J., Walsh E., Bollina P., Catto J., Doble A., Doberty A., Gillatt D., Kockelbergh R., Kynaston H., Paul A., Powell P., Prescott S., Rosario D.J., Rowe E., Neal D.E.; ProtecT Study Group. 10-Year outcomes after monitoring, surgery, or radiotherapy for localized prostate cancer. N Engl J Med. 2016 Oct 13; 375(15): 1415-1424.

20. Donovan J.L., Hamdy F.C., Lane J.A., Mason M., Metcalfe C., Walsh E., Blazeby J.M., Peters T.J., Holding P., Bonnington S., Lennon T., Bradshaw L., Cooper D., Herbert P., Howson J., Jones A., Lyons N., Salter E., Thompson P., Tidball S., Blaikie J., Gray C., Bollina P., Catto J., Doble A., Doherty A., Gillatt D., Kockelbergh R., Kynaston H., Paul A., Powell P., Prescott S., Rosario D.J., Rowe E., Davis M., Turner E.L., Martin R.M., Neal D.E.; ProtecT Study Group. Patient-reported outcomes after monitoring, surgery, or radiotherapy for prostate cancer. N Engl J Med. 2016 Oct 13; 375(15): 1425-1437.

21. Liang K., Ang K.K., Milas L., Hunter N., Fan Z. The epidermal growth factor receptor mediates radioresistance. Int J RadiatOncolBiol Phys. 2003; 57: 246-254. PMID: 12909240.

22. Akimoto T., Hunter N.R., Buchmiller L., Mason K., Ang K.K., Milas L. Inverse relationship between epidermal growth factor receptor expression and radiocurability of murine carcinomas. Clin Cancer Res. 1999; 5: 2884-2890. PMID: 10537357.

23. Khalifa J., Amini A., Popat S., Gaspar L.E., Faivre-Finn C. Brain metastases from NSCLC: radiation therapy in the era of targeted therapies. Journal of Thoracic Oncology 2016; 11(10): 1627-1643.

24. Spigel D.R., Hainsworth J.D., Yardley D.A., et al. Tracheoesophageal fistula formation in patients with lung cancer treated with chemoradiation and bevacizumab. J ClinOncol 2010; 28: 43-8. doi: 10.1200/JCO.2009.24.7353. Epub 2009 Nov 9. PMID: 19901100.

25. Kachnic L.A., Moughan J., Suntharalingam M., Ilson D.H., Konski A.A., Burrows W., Anker C., Bar Ad V., Thakrar H.V., Hayes J.P., Gore E.M., Kavadi V.S., Komaki R.U., Raben A., Giguere J.K., Pollock J., Greenberger J.S., Videtic G.M., Roof K.S., Watkins Bruner D. Patient-reported outcomes (PROs) in NRG Oncology/RTOG 0436: a Phase 3 trial evaluating the addition of cetuximab to paclitaxel, cisplatin, and radiation for esophageal cancer treated without surgery. Int $\mathrm{J}$ RadiatOncolBiol Phys 2016, 96(2): S31.

26. Gan G.N., Weickhardt A.J., Scheier B., Doebele R.C., Gaspar L.E., Kavanagh B.D., Camidge D.R. Stereotactic radiation therapy can safely and durably control sites of extra-central nervous system oligoprogressive disease in anaplastic lymphoma kinase-positive lung cancer patients receiving crizotinib. Int J RadiatOncolBiol Phys. 2014 Mar 15; 88(4): 892-8.

27. Luo S., Chen L., Chen X., Xie X. Evaluation on efficacy and safety of tyrosine kinase inhibitors plus radiotherapy in NSCLC patients with brain metastases. Oncotarget. 2015; 6: 16725-16734. doi: 10.18632/oncotarget.4264. PMID: 26057469 .

28. Lee Y., Aub S.L., Wang Y., Burnette B., Wang Y., Meng Y., Beckett M., Sharma R., Chin R., Tu T., Weichselbaum R.R., $F u Y . X$. Therapeutic effects of ablative radiation on local tumor require CD8+ T cells: changing strategies for cancer treatment. Blood. 2009 Jul 16; 114(3): 589-95. 
29. Stone H.B., Peters L.J., Milas L. Eff ect of host immune capability on radiocurability and subsequent transplantability of a murine fi brosarcoma. J Natl Cancer Inst 1979; 63: 1229-35. PMID: 291749.

30. Siva S., Callaban J., MacManus M.P., Martin O., Hicks R.J., Ball D.L. Abscopal effects after conventional and stereotactic lung irradiation of non-small-cell lung cancer. Journal of Thoracic Oncology 2013; 8(8): e71-72.

31. Tao R., Krishnan S., Bhosale P.R., Javle M.M., Aloia T.A., ShroffR.T., Kaseb A.O., Bishop A.J., Swanick C.W., Koay E.J., Thames H.D., Hong T.S., Das P., Crane C.H. Ablative radiotherapy doses lead to a substantial prolongation of survival in patients with inoperable intrahepatic cholangiocarcinoma: a retrospective dose response analysis. J ClinOncol 2016; 34: 219-226. doi: 10.1200/JCO.2015.61.3778. Epub 2015 Oct 26. PMID: 26503201.

32. Wabl D.R., Stenmark M.H., Tao Y., Pollom E.L., Caoili E.M., Lawrence T.S., Schipper M.J., Feng M. Outcomes after stereotactic body radiotherapy or radiofrequency ablation for hepatocellular carcinoma. J ClinOncol. 2016; 34(5): 452-9. doi: 10.1200/JCO.2015.61.4925. Epub 2015 Nov 30. PMID: 26628466.

33. Hong T.S., Wo J.Y., Yeap B.Y., Ben-Josef E., McDonnell E.I., Blaszkowsky L.S., Kwak E.L., Allen J.N., Clark J.W., Goyal L., Murphy J.E., Javle M.M., WolfgangJ.A., Drapek L.C., Arellano R.S., Mamon H.J., Mullen J.T., Yoon S.S., Tanabe K.K., Ferrone C.R., Ryan D.P., DeLaney T.F., Crane C.H., Zhu A.X. Multi-institutional phase ii study of high-dose hypofractionated proton beam therapy in patients with localized, unresectable hepatocellular carcinoma and intrahepatic cholangiocarcinoma. J ClinOncol 2016; 34: 460-468. doi: 10.1200/JCO.2015.64.2710. Epub 2015 Dec 14. PMID: 26668346. 